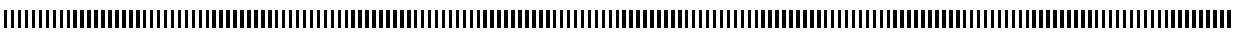

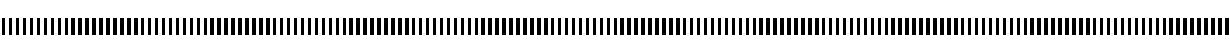

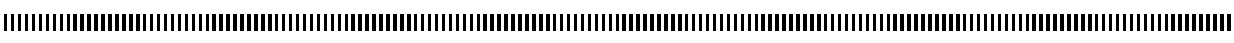

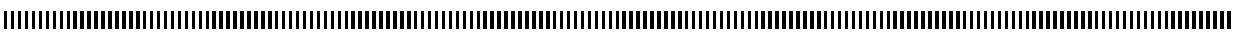

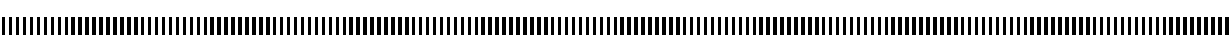

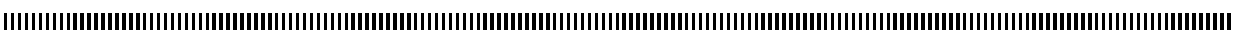

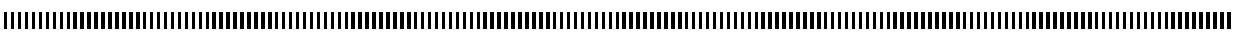

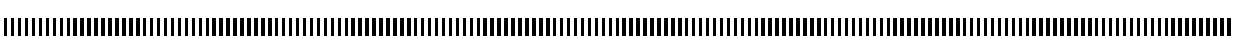

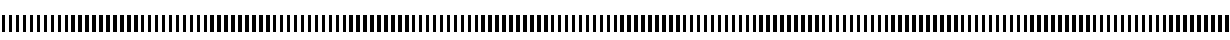

\title{
Canard-induced loss of stability across a homoclinic bifurcation
}

\author{
Mathieu Desroches* — Jean-Pierre Françoise ${ }^{* *}$ — Lucile Mégret*** \\ * INRIA Paris-Rocquencourt Research Centre, MYCENAE Project-Team \\ Domaine de Voluceau BP 105, 78153 Le Chesnay cedex, France \\ mathieu.desroches@inria.fr \\ ** Université P.-M. Curie, Paris 6, Laboratoire Jacques-Louis Lions \\ UMR 7598 CNRS, 4 Place Jussieu, 75252, Paris Cedex, France \\ Jean-Pierre.Francoise@upmc.fr \\ *** Université P.-M. Curie, Paris 6, Laboratoire Jacques-Louis Lions \\ UMR 7598 CNRS, 4 Place Jussieu, 75252, Paris Cedex, France \\ lucile.megret@ann.jussieu.fr
}

\begin{abstract}
|
ABSTRACT. This article deals with slow-fast systems and is, in some sense, a first approach to a general problem, namely to investigate the possibility of bifurcations which display a dramatic change in the phase portrait in a very small (on the order of $10^{-7}$ in the example presented here) change of a parameter. We provide evidence of existence of such a very rapid loss of stability on a specific example of a singular perturbation setting. This example is strongly inspired of the explosion of canard cycles first discovered and studied by E Benoît, J.-L. Callot, F. Diener and M. Diener. After some presentation of the integrable case to be perturbed, we present the numerical evidences for this rapid loss of stability using numerical continuation. We discuss then the possibility to estimate accurately the value of the parameter for which this bifurcation occurs.
\end{abstract}

RÉSUMÉ. Cet article traite de systèmes lents-rapides et constitue en quelque sorte une première approche pour étudier un problème général, celui d'explorer les possibilités de bifurcations qui présentent un changement brutal au niveau du portrait de phase pour une très petite variation de paramètre (de l'ordre de $10^{-7}$ dans l'exemple présenté ici). Nous donnons des preuves de l'existence d'une perte brutale de stabilité de ce type sur un exemple spécifique dans un cadre de perturbations singulières. Cet exemple est fortement inspiré de l'explosion de cycles canards initialement découverte par E. Benoît, J.-L. Callot, F. Diener et M. Diener. Après une présentation du cas intégrable que l'on souhaite perturber, nous apportons une preuve numérique de cette perte brutale de stabilité obtenue en utilisant une méthode de de continuation numérique. Nous discutons ensuite de la possibilité d'estimer précisément la valeur de paramètre pour laquelle cette bifurcation se produit.

KEYWORDS : slow-fast systems, canard solutions, Lambert function, numerical continuation

MOTS-CLÉS : systèmes lents-rapides, solutions canard, fonction de Lambert, continuation numérique

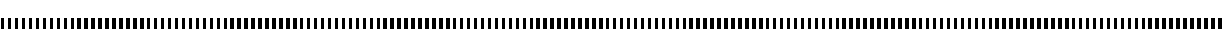




\section{Introduction}

Hopf bifurcation has been thoroughly studied in two dimensional vector fields with one slow and one fast variable; see for instance [6, 15, 19, 23, 24, 29]. This singular version of the Hopf bifurcation displays an explosion of cycles as the periodic orbit which emerges from the Hopf bifurcation grows explosively from an amplitude that is $O\left(\varepsilon^{1 / 2}\right)$ to an amplitude that is $O(1)$ over a range of values of the effective parameter $\mu$ of length $O(\exp (-c / \varepsilon))$. This phenomenon involves limit cycles named canards and which have the peculiar property to follow both attracting and repelling (locally) invariant manifolds. The explosion of canard cycles has first been detected and studied using non-standard analysis [5, 6] and asymptotic and numerical analysis [2, 3, 19]. A description by means of geometric singular perturbation theory, has been provided for the Van der Pol case in [15]. A treatment of a more general case using the techniques introduced in [15] was later developped in [29]. The normal form aspect was shown in [7]. J. Guckenheimer developed a canonical system for singular Hopf bifurcation with two slow variables in several articles $[24,25]$. We consider here the case of one slow variable and analyse a more general family which involves a global perturbation setting. In this context, one can carefully keep track of periodic orbits by using both analytical and numerical continuation methods. The combination of canard explosion and homoclinic bifurcation has been investigated in various frameworks, both in singularly perturbed ODEs [4, 10, 27, 30] and in context of traveling wave solutions of PDEs [8, 26].

\section{Theoretical importance of canard cycles in the perturbation theory of periodic orbits}

Relaxation oscillations are represented by limit cycles which appear in slow-fast systems at the boundary of classical polynomial Liénard equations:

$$
\varepsilon x^{\prime \prime}+f(x) x^{\prime}+x=\lambda,
$$

where $f(x)$ is a polynomial. To this equation, one associates the planar vector field:

$$
\begin{aligned}
& x^{\prime}=y-F(x) \\
& y^{\prime}=-\varepsilon(x-\lambda),
\end{aligned}
$$

where $F(x)=\int_{0}^{x} f(s) d s$. For $\varepsilon=0$ we have a family of layer equations whose critical curves are given by $y=F(x)$. A slow-fast cycle of a layer equation is a simple closed curve, union of regular trajectories (horizontal arcs in the complement of the critical curve) and arcs on the slow curve (critical arcs) which is a limit-periodic set, from which limit cycles bifurcate when $\varepsilon>0$ is small enough. Critical arcs are either attracting or repelling depending of the sign of $F^{\prime}(x)$. A slow-fast cycle is called common if all its 
critical arcs are of same type. Such a slow-fast cycle bifurcates when $\varepsilon \rightarrow 0$ into a single hyperbolic cycle. If a slow-fast cycle contains critical arcs of different type, it is called a canard cycle.

In [20], an example was given of an attracting limit-periodic set that is a polycycle with an attractive and a repulsive sector, which bifurcates into multiple limit cycle. Canard cycles can, for the same reason, bifurcate in multiple limit cycles. This allows to find more limit cycles than expected in Liénard equation $[11,16]$.

In relation with the study of the Hilbert 16th problem, it is important to further develop global bifurcation theory inside the class of generalized polynomial Liénard system of type $(m, n)$ :

$$
\begin{aligned}
& \varepsilon \dot{x}=y-\frac{1}{2} x^{2}+\sum_{i=0}^{n+1} \alpha_{i}(\varepsilon) x^{i}, \\
& \dot{y}=-x+\varepsilon \sum_{j=1}^{m} \beta_{j}(\varepsilon) x^{j},
\end{aligned}
$$

where $\varepsilon$ is a small parameter, and $\alpha_{i}$ and $\beta_{j}$ are bounded parameters.

\section{A general perturbation setting}

We propose to study pertubation theory of the equation:

$$
\begin{aligned}
& \varepsilon \dot{x}=y-f(x) \\
& \dot{y}=-f^{\prime}(x),
\end{aligned}
$$

where $f(x)$ is a polynomial. We assume that all critical points of $f(x)$ (zeros of $f^{\prime}(x)$ ) are simple. After translation of coordinates, we can assume that one of the minima of $f(x)$ is at the origin and thus $f(x)=\frac{x^{2}}{2}+O\left(x^{3}\right)$. By a scaling time and variables appropriately,

$$
(x, y, t) \mapsto\left(\varepsilon^{1 / 2} x, \varepsilon y, \varepsilon^{1 / 2} t\right),
$$

the equation is transformed into

$$
\begin{aligned}
& \dot{x}=y-f_{\varepsilon}(x) \\
& \dot{y}=-f_{\varepsilon}^{\prime}(x) .
\end{aligned}
$$

This equation is integrable and it possesses the first integral:

$$
H_{\varepsilon}:(x, y) \mapsto H_{\varepsilon}(x, y)=\mathrm{e}^{-y}\left[f_{\varepsilon}(x)-y-1\right] .
$$

In the initial coordinates, we find the first integral

$$
H_{\varepsilon}:(x, y) \mapsto H_{\varepsilon}(x, y)=\mathrm{e}^{-y / \varepsilon}\left[\frac{f(x)-y}{\varepsilon}-1\right] .
$$


This family of integrable slow-fast systems is particularly interesting because there is both an explicit form of the first integral and furthermore the slow curve is explicitely defined as $\{y=f(x)-\varepsilon\}$.

Lemma 1. The critical points of the function $H$ are exactly the points $\left(x_{0}, y_{0}\right)$ such that $f^{\prime}\left(x_{0}\right)=0$ and $y_{0}=f\left(x_{0}\right)$. Critical values of $H$ are $h_{0}=-\exp \left(-f\left(x_{0}\right) / \varepsilon\right)<0$. The critical points of $H$ are Morse points either of center type (for local minima of $f(x)$ ) or of saddle type (for local maxima of $f(x)$ ).

Proof. If we choose local coordinates centred at the points $\left(x_{0}, y_{0}=f\left(x_{0}\right)\right.$ :

$$
\begin{aligned}
& x=x_{0}+X \\
& y=f\left(x_{0}\right)+Y,
\end{aligned}
$$

The Taylor expansion of $H_{\varepsilon}$ at the point $\left(x_{0}, y_{0}\right)$ yields:

$$
H_{\varepsilon}(X, Y)=\mathrm{e}^{-\frac{f\left(x_{0}\right)}{\varepsilon}}\left[-1+\frac{f^{\prime \prime}\left(x_{0}\right)}{2 \varepsilon} X^{2}+\frac{2}{\varepsilon^{2}} Y^{2}+\ldots\right]
$$

and this shows the lemma.

Note that closed orbits of the Hamiltonian associated with $H$ cannot intersect the slow curve (which is contained in $H=0$ ). As they must contain a critical point of $H$ in their interior, they are necessarily all above the slow curve, in the domain union of $\{H=h<$ $0\}$. Furthermore, the Morse lemma shows that all orbits close to a critical point associated to a minima of $f$ are necessarily closed. The phase portrait of the Hamiltonian of $H$ displays nested periodic orbits around each critical points of center type. The topological type of the level sets of $\{H=h\}$ can change only when $h$ crosses a critical value. Such critical levels corresponds to homoclinic or heteroclinic loops. In some cases, a nest of periodic orbits bounded by a heteroclinic loop can be included in another nest of periodic orbits as seen below.

To simplify the drawings, we choose to represent the level sets of $H$ for $\varepsilon=1$ in the example:

$$
\begin{aligned}
\varepsilon \dot{x} & =y-f(x) \\
\dot{y} & =-f^{\prime}(x) \\
\text { with : } f(x) & =(x+1) x(x-1)\left(x-\frac{3}{2}\right)(x-3) .
\end{aligned}
$$




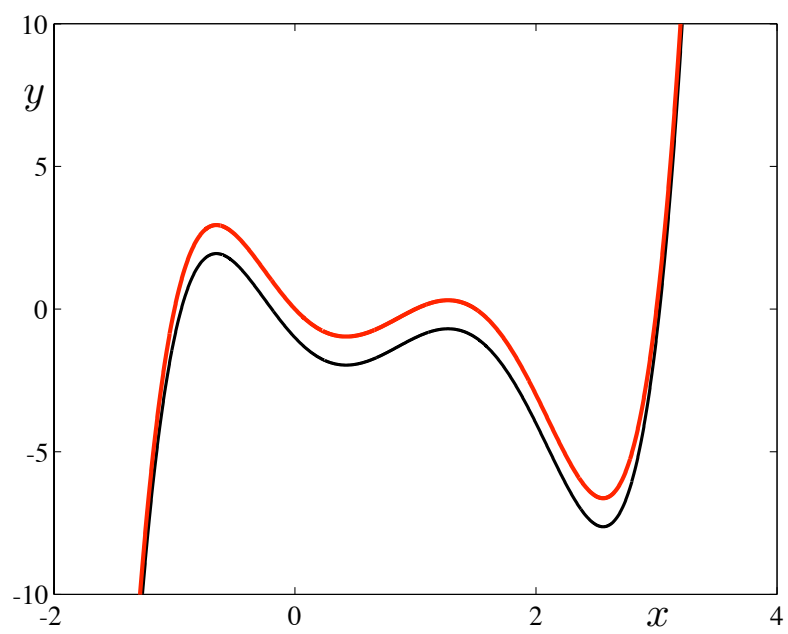

Figure 1. Slow curve $\{y=f(x)-1\}$ (in black) and critical curve (in red).

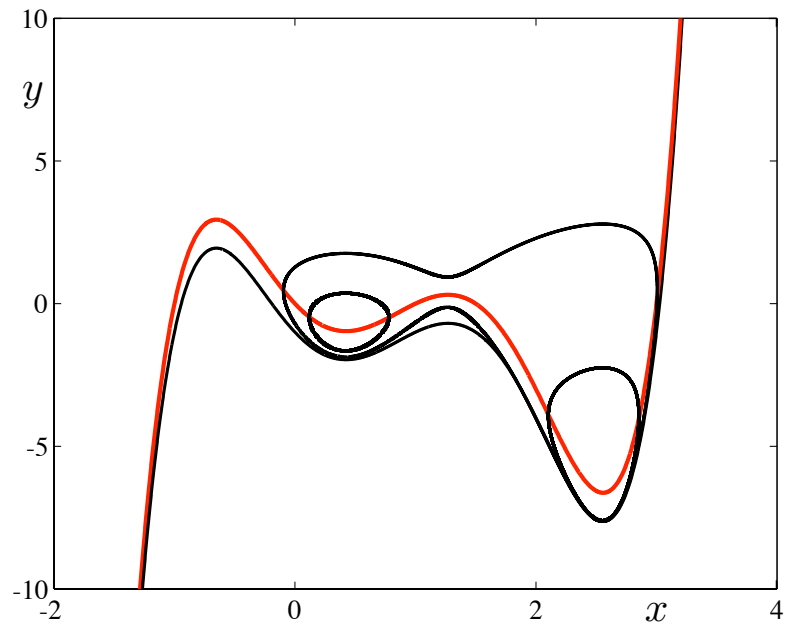

Figure 2. Three cycles belonging to three different nests. 


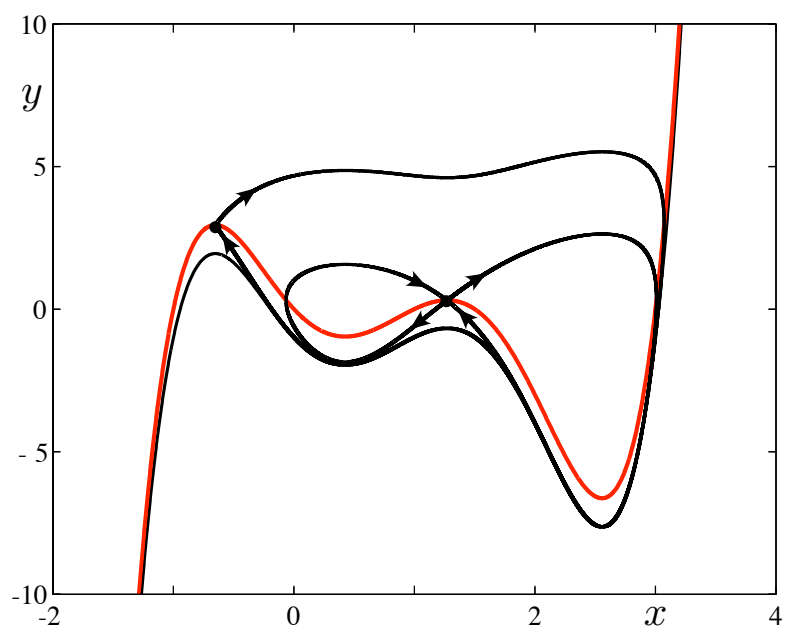

Figure 3. The three homoclinic loops which bound each nests.

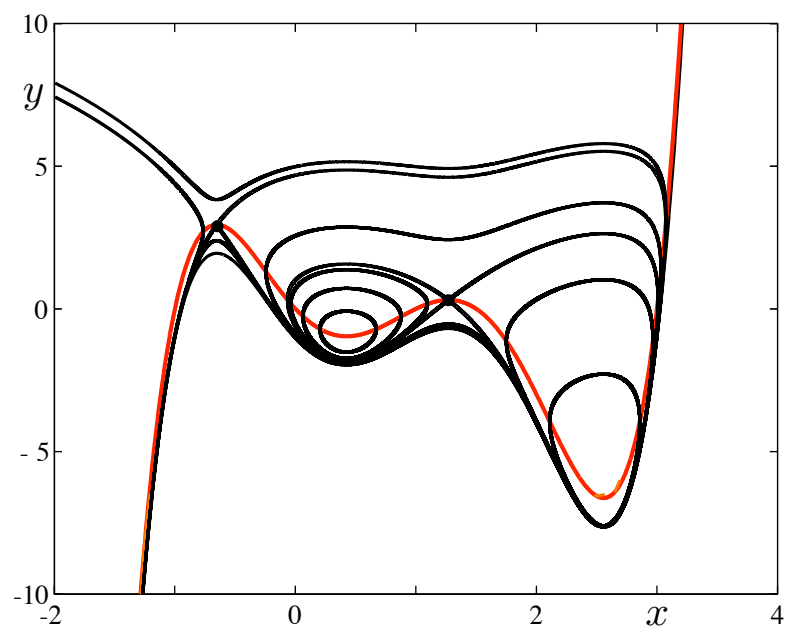

Figure 4. Full phase portrait. 
Consider next this second example

$$
\begin{aligned}
\varepsilon \dot{x} & =y-f(x) \\
\dot{y} & =-f^{\prime}(x) \\
\text { with : } f(x) & =\frac{x^{2}}{2}-\frac{x^{4}}{4} .
\end{aligned}
$$

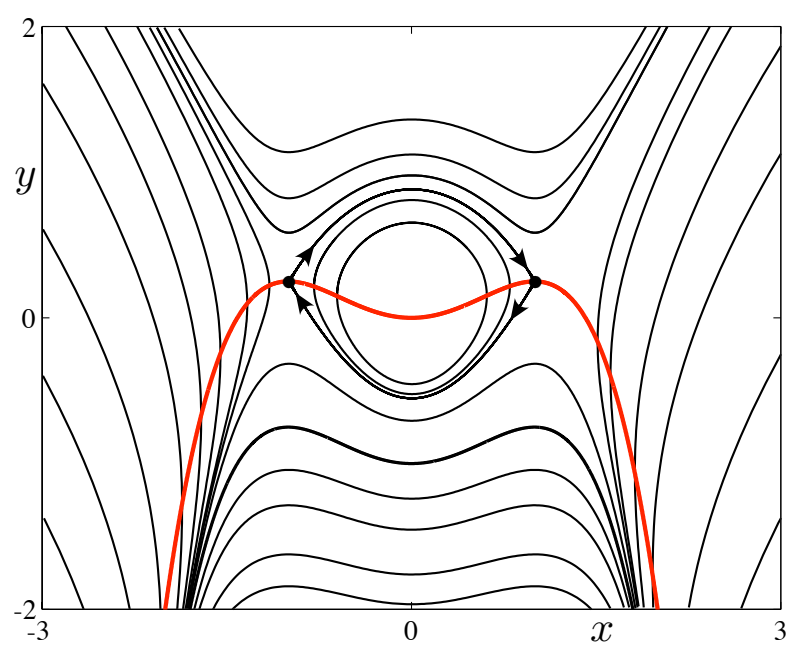

Figure 5. A nest bounded by a heteroclinic loop

\section{The Lambert function and the parametrization of the level sets of $H$}

Consider the entire function:

$$
f: w \mapsto z=w \mathrm{e}^{w} .
$$

This function is locally invertible near $w=0$ and the Lagrange inversion theorem yields as inverse the series:

$$
W_{0}(z)=\sum_{n=1}^{+\infty} \frac{(-n)^{n-1}}{n !} z^{n} .
$$

This convergent series defines the so-called principal branch of the Lambert function [9].

Note that $f$ displays a critical point at $w=-1$, hence a critical value at $z=-1 / \mathrm{e}$. This implies that the radius of convergence of the series is less than or equal to $1 / \mathrm{e}$. In 
fact, it is exactly equal to $1 / \mathrm{e}$, which is easily seen using the ratio test. The following lemma can be proved (see [9] p. 23). For the reader's convenience, we recall the proof.

Lemma 2. The Lambert function displays a square root branching point at the point $z=-1 / \mathrm{e}$. More precisely, if we change variable $z$ into $p=\sqrt{2(\mathrm{e} z+1)}$, then there exists a convergent series on the disc $p \in D(0, \sqrt{2})$ such that:

$$
W_{0}(z)=-1+\Phi(p)=-1+p-\frac{1}{3} p^{2}+\frac{11}{72} p^{3}+\ldots
$$

Proof. Write $z=w \mathrm{e}^{w}$, with $w=-1+u$. This displays $w=\frac{1}{\mathrm{e}}(-1+u) \mathrm{e}^{u}$, hence:

$$
z=\frac{1}{\mathrm{e}}\left[-1+\sum_{k \geq 2}\left(\frac{1}{(k-1) !}-\frac{1}{k !}\right) u^{k}\right] .
$$

If $p=\sqrt{2(\mathrm{e} z+1)}$,

$$
z=\frac{p^{2}}{2}-1=-1+\frac{u^{2}}{2}+\ldots
$$

By the local inversion theorem, this yields two possible analytical branches:

$$
\begin{aligned}
& u=\Phi(p)=p+\ldots \\
& u=\Phi(-p)=-p+\ldots
\end{aligned}
$$

Note that the values $W_{0}(z)>-1, z \in \mathbb{R}$ thus $W_{0}(z)=-1+\Phi(p)$ and the other choice defines another real branch of the Lambert function that is denoted as $W_{-1}$ :

$$
\begin{aligned}
& W_{0}(z)=-1+\Phi(p) \\
& W_{-1}(z)=-1+\Phi(-p)
\end{aligned}
$$

The square-root branching point displayed by the main branch of the Lambert function $W_{0}(z)$ gives birth to another branch $W_{-1}(z)$ which also solves the equation $w \mathrm{e}^{w}=z$. There are indeed infinitely many solutions in the complex to the equation and correspondingly infinitely many complex branches of the Lambert functions. Note that this whole complex ramification was preceedingly used in relation with a family of Abel equation in [21]. Here we focus only on the two branches $W_{0}$ and $W_{-1}$ which are the only branches which take real values over the real.

Consider the slow-fast system defined previously:

$$
\begin{aligned}
& \varepsilon \dot{x}=y-f(x) \\
& \dot{y}=-f^{\prime}(x) .
\end{aligned}
$$

System (20) displays the first integral 


$$
e^{-y / \varepsilon}\left[\frac{f(x)-y}{\varepsilon}-1\right]=h=\text { constant } .
$$

Thus trajectories can be split in two parts

$$
\begin{aligned}
& y_{+}=f(x)-\varepsilon-\varepsilon W_{-1}\left(\frac{h}{e} \mathrm{e}^{f(x) / \varepsilon}\right), \\
& y_{-}=f(x)-\varepsilon-\varepsilon W_{0}\left(\frac{h}{e} \mathrm{e}^{f(x) / \varepsilon}\right) .
\end{aligned}
$$

To complete the description of the periodic of these systems, we prove the following proposition:

Proposition 3. Any periodic trajectory intersects transversally the critical curve in exactly two points.

Proof. Consider first a fast trajectory and assume that it could be tangent to the critical curve. This would yield to two equations

$$
\begin{aligned}
& W\left(\frac{h}{e} \mathrm{e}^{f(x) / \varepsilon}\right)=1, \\
& W^{\prime}\left(\frac{h}{e} \mathrm{e}^{f(x) / \varepsilon}\right)\left(\frac{h}{e} \mathrm{e}^{f(x) / \varepsilon}\right) f^{\prime}(x) / \varepsilon=0 .
\end{aligned}
$$

This implies both $y=f(x)$ and $f^{\prime}(x)=0$ and so this fast trajectory must contain a stationary point. Consider now a periodic orbit. Its fast part cannot contain a stationary point and hence it intersects the critical curve transversally. Assume it would intersect the critical curve in more than two points (hence at least 4 points). Then there would be a contradiction with the constant sign of $\dot{x}$ above of the critical curve. So the fast part of the periodic orbit intersects the critical curve in two points. It is then easily checked that on the left and on the right of these intersection points the periodic orbit extends with a slow part which goes between the critical curve and the slow curve $y=f(x)-\varepsilon$ without possible further intersection with the critical curve. The two parts of the periodic orbit, slow and fast, correspond exactly to the two parametrizations of the level sets of $H$ with $W_{-1}$ and $W_{0}$ respectively.

\section{An example of canard-induced loss of stability}

We consider the particular case of integrable Liénard equations (depending of a parameter $\alpha$ ):

$$
\begin{aligned}
\varepsilon \dot{x} & =y-\frac{x^{2}}{2}-\alpha \frac{x^{3}}{3} \\
\dot{y} & =-x-\alpha x^{2},
\end{aligned}
$$

and the pertubation setting (depending of two more parameters $\beta, \mu$ ):

$$
\begin{aligned}
\varepsilon \dot{x} & =y-\frac{x^{2}}{2}-\alpha \frac{x^{3}}{3} \\
\dot{y} & =-x-x^{2}(\alpha-\beta)+\sqrt{\varepsilon} \mu .
\end{aligned}
$$


After rescaling this yields:

$$
\begin{aligned}
& \dot{x}=y-\frac{x^{2}}{2}-\sqrt{\varepsilon} \alpha \frac{x^{3}}{3} \\
& \dot{y}=-x-\sqrt{\varepsilon} x^{2}(\alpha-\beta)+\mu .
\end{aligned}
$$

Numerical simulations have been done with XPPAUT [18]. They are shown on the two panels of Fig. 8. For $\alpha=0.395, \sqrt{\varepsilon}=0.03125$ and $\beta=0.01$, a small canard cycle is born for $\mu$ very small and negative. Then for $\mu$ between -0.0003128 and -0.0003127 , there is a cycle explosion where the small cycle jumps to the maximal cycle which disappears across an homoclinic bifurcation. In order to capture the entire bifurcation sequence, from the Hopf bifurcation to the homoclinic bifurcation via the canard explosion, we have used numerical continuation with the software package AUTO. Path-following methods [28], used in conjunction with boundary-value solvers [12,13], are very effective to treat numerically multiple-timescale problems, which are very stiff due to strong repulsion in the normal direction to slow manifolds, hence particularly in the canard regime; see [11] for more details on this topic. To illustrate this point, we show on Fig. 6 the bifurcation diagram of system (26) with respect to $\mu$ (panel (a)) as well as a selection of canard cycles approaching the (canard) homoclinic connection (panel (b)).

(a)

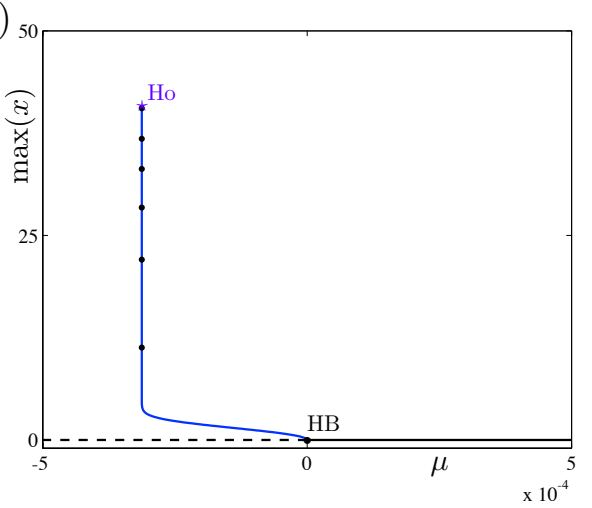

(b)

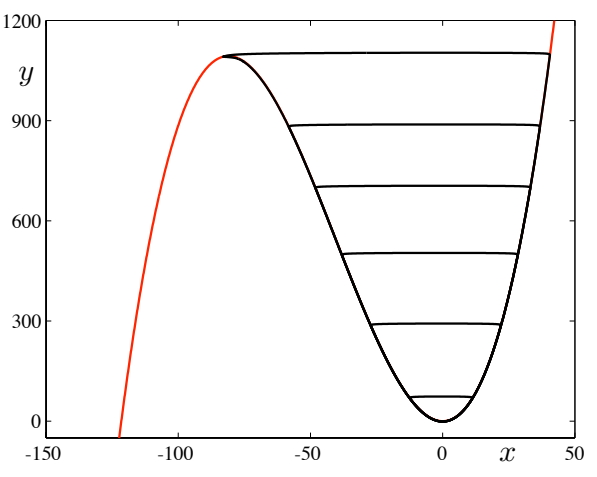

Figure 6. (a): Bifurcation diagram of system (26) in $\mu$. (b): a few limit cycles on the explosive branch (in blue) shown in panel (a), approaching the homoclinic connection. 
(a)

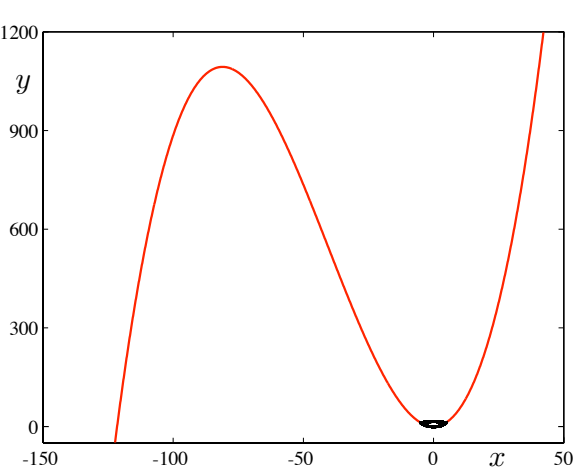

(b)

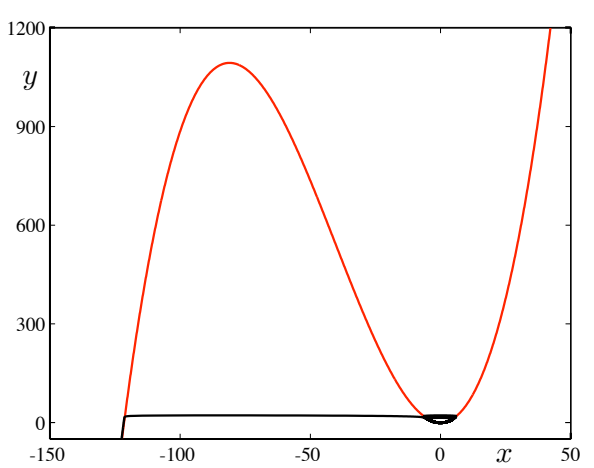

Figure 7. (a): Small canard cycle for $\alpha=0.395, \sqrt{\varepsilon}=0.03125, \beta=0.01$ and $\mu=$ -0.0003127 . (b): Trajectory with same initial condition for $\mu=-0.0003128$.

We propose to try to estimate the bifurcation value of the parameter $\mu$ using the traditional approach to cycle explosion based on asymptotics. We closely follow estimates proposed by Eckhaus for the case of the van der Pol equation. There are good reasons to believe that this approach could be adapted because we have in the integrable case an explicit equation for the slow curve.

It is convenient to write the perturbed system as

$$
\begin{array}{rlll}
\varepsilon \dot{x} & =y-f(x) & = & y-\frac{x^{2}}{2}-\alpha \frac{x^{3}}{3} \\
\dot{y} & =-f^{\prime}(x)+\delta(x) & = & -x-\alpha x^{2}+\sqrt{\varepsilon} \mu+\beta x^{2} .
\end{array}
$$

Note that for the unperturbed case, $\delta(x)=0, y-f(x)=-\varepsilon$ is a solution (slow curve). In the perturbed case, $\delta(x) \neq 0$, we can look for a solution $\phi(x, \varepsilon)$ of the equation:

$$
y-f(x)=-\varepsilon+\varepsilon^{2} \phi(x, \varepsilon),
$$

defined on an interval $\left[x_{0}, x_{1}\right]$ so that $\left|x_{0}\right| \leq \frac{1}{\alpha}$.

As $\dot{x}=-1+\varepsilon \phi$ and $\varepsilon \dot{\phi}=-\frac{\delta(x)}{\varepsilon}-\phi f^{\prime}(x)$, the unknown function $\phi$ must be solution of the differential equation:

$$
\varepsilon[-1+\varepsilon \phi] \frac{d \phi}{d x}=-f^{\prime}(x) \phi+\frac{\delta(x)}{\varepsilon} .
$$

This equation can be cast into:

$$
\begin{aligned}
\phi^{\prime}(x) & =\left[\frac{f^{\prime}(x)}{\varepsilon}+\varepsilon \phi^{\prime}(x)\right] \phi(x)-\frac{\delta(x)}{\varepsilon^{2}} \\
& =\left[\frac{f(x)}{\varepsilon}+\varepsilon \phi(x)\right]^{\prime} \phi(x)-\frac{\delta(x)}{\varepsilon^{2}} .
\end{aligned}
$$


Following Eckaus, to solve this equation, we first solve the associated homogeneous equation:

$$
\phi^{\prime}(x)=\left[\frac{f(x)}{\varepsilon}+\varepsilon \phi(x)\right]^{\prime} \phi(x) .
$$

This yields,

$$
\phi(x)=K \exp \left(\frac{f(x)-f\left(x_{0}\right)}{\varepsilon}+\varepsilon\left(\phi(x)-\phi\left(x_{0}\right)\right)\right) .
$$

By a classical method of variation of the constant, we obtain the solution of equation 30 as follows:

$$
\begin{aligned}
\phi(x) & =e^{\frac{Q(x)}{\varepsilon}}\left[\phi\left(x_{0}\right) e^{-\frac{Q\left(x_{0}\right)}{\varepsilon}}-\frac{1}{\varepsilon^{2}} \int_{x_{0}}^{x} e^{-\frac{Q(t)}{\varepsilon}} \delta(t) d t\right] \\
Q(x) & =f(x)+\varepsilon^{2} \phi(x) .
\end{aligned}
$$

Following again the approach of Eckhaus, we find that there exists a bounded solution $\phi$ for

$$
\begin{aligned}
\beta & =O(\varepsilon) \\
\mu & =\mu_{c}+\varepsilon^{5 / 2} \sigma e^{-\frac{k^{2}}{\varepsilon}} \\
\mu_{c} & =-\sqrt{\varepsilon} \beta(1+d)[1+o(1)]
\end{aligned}
$$

for some $d \in[-2 / 3,2 / 3]$. This approximation is consistent with the parameter interval in which canard solutions are found numerically. Indeed, we find by direct numerical simulation with XPPAUT that canards exist for $\mu$ approximately in the interval $]-0.0003128,0[$. This approximation matches what is obtained with a better precision by using numerical continuation with AUTO; see Fig. 6 (a). Moreover, we observe that the numerical lower bound is consistent with the theoretical value

$$
\mu_{c}=-\sqrt{\varepsilon} \beta=-0.0003125 .
$$

If we reproduce Eckhaus' analysis for the van der Pol system

$$
\begin{aligned}
\varepsilon \dot{x} & =y-\frac{x^{2}}{2}-\alpha \frac{x^{3}}{3} \\
\dot{y} & =-x+\sqrt{\varepsilon} \mu
\end{aligned}
$$

we find that

$$
\begin{aligned}
\mu & =\mu_{c}+\varepsilon^{3 / 2} \sigma e^{-\frac{k^{2}}{\varepsilon}}, \\
\mu_{c} & =\sqrt{\varepsilon} \alpha(1+o(1)) .
\end{aligned}
$$

This matches what we obtain by numerical simulation with XPPAUT. 
(a)

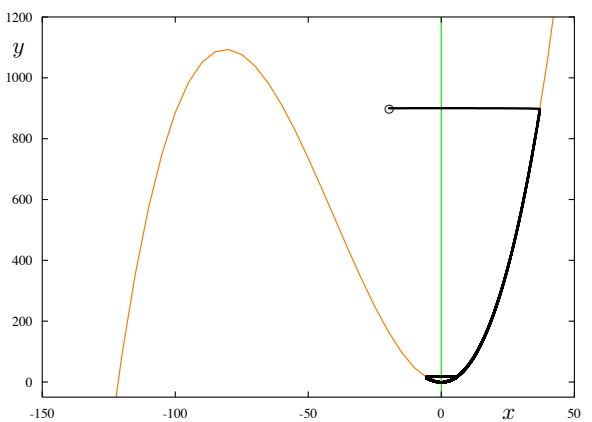

(b)

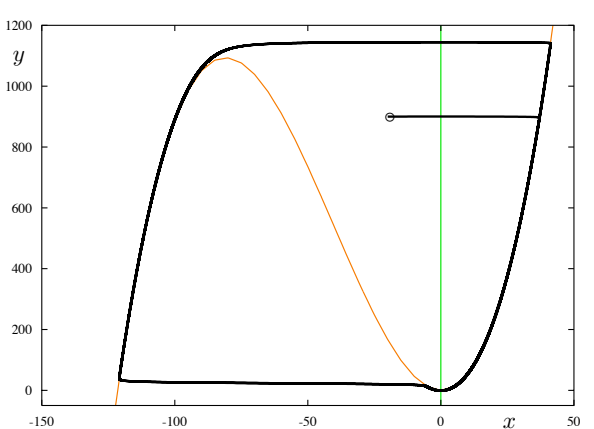

Figure 8. Canards in the classical van der Pol equation (35). Panel(a): Small canard cycle for $\alpha=0.395, \sqrt{\varepsilon}=0.03125$ and $\mu=-0.0123664$. Panel (b): Large canard cycle for $\mu=-0123665$.

We also verify numerically that the amplitude of the parameter interval in which canards exist is well approximated by the value $\sqrt{\varepsilon} \alpha=0.0123438$.

This first approach gives a first approximation of the parameter interval in which canard solutions exist but it is not clear that it allows to follow the canard cycle until it reaches the homoclinic bifurcation. Indeed in the case of the van der Pol system Eckhaus' analysis is not valid close to the point satisfying $1+\alpha x=0$. It seems that in the case of system eq:sytst we do not have this restriction. An interesting fact is that the parameter interval in which canard solutions exist in the generalized Lambert system (26) is very small.

We now want to predict for which parameter value the homoclinic bifurcation occurs. This justifies to develop another strategy based on the first-return map and its first derivative given by an integral of Lambert functions.

Consider the equation

$$
\begin{aligned}
h & =e^{-\frac{y}{\varepsilon}}\left[\frac{f(x)}{\varepsilon}-\frac{y}{\varepsilon}-1\right] \\
\omega & =e^{-\frac{y}{\varepsilon}} \frac{y-f(x)}{\varepsilon} d y-e^{-\frac{-y}{\varepsilon}}\left(-f^{\prime}(x)-\delta(x)\right) d x \\
& =d h-e^{-\frac{y}{\varepsilon}} \delta(x) d x
\end{aligned}
$$

Consider the solution of the full equation passing by the point $\left(0,-\varepsilon\left[1+W_{-1}\left(h e^{-1}\right)\right]\right)$. Let $L_{+}(h, \beta, \mu)$ be the (y-value) first intersection of this trajectory with the axis $\{x=0\}$ following the positive flow. Let $L_{-}(h, \beta, \mu)$ be the intersection point with $\{x=0\}$ of this trajectory following the negative flow. Let $\gamma_{\mu, \beta, h}$ be the closed arc union of the 
arc of trajectory between the two points $L_{+}(h, \beta, \mu)$ and $L_{-}(h, \beta, \mu)$ and the segment $\left[L_{-}(h, \beta, \mu), L_{+}(h, \beta, \mu)\right]$ on the axis $x=0$. Then, the following integral:

$$
\int_{\gamma_{\mu, \beta, h}} \omega=\int_{\gamma_{\mu, \beta, h}} d h-\int_{\gamma_{\mu, \beta, h}} e^{-\frac{y}{\varepsilon}} \delta(x) d x,
$$

displays the condition:

$$
L_{+}(h, \mu, \beta)-L_{-}(h, \mu, \beta)=-\int_{\gamma_{0}} e^{-\frac{y}{\varepsilon}} \delta(x) d x+O\left((\sqrt{\mu}, \beta)^{2}\right) .
$$

Recall the parametrization of the level sets of the Hamiltonian

$$
\begin{aligned}
y(x) & =f(x)-\varepsilon\left[1+W\left(\frac{h}{e} e^{\frac{f(x)}{\varepsilon}}\right)\right], \\
e^{W(u)} & =\frac{u}{W(u)}
\end{aligned}
$$

for $W=W_{-1}$ or $W=W_{0}$. This displays:

$$
\begin{aligned}
\frac{L_{+}(h, \beta, \mu)-L_{-}(h, \beta, \mu)}{h}= & \beta \underbrace{\int_{x^{-}(h)}^{x^{+}(h)} x^{2}\left[\frac{1}{W_{0}\left(\frac{h}{e} e^{\frac{f(x)}{\varepsilon}}\right)}-\frac{1}{W_{-1}\left(\frac{h}{e} e^{\frac{f(x)}{\varepsilon}}\right)}\right] d x}_{I_{2}(h)} \\
& +\sqrt{\varepsilon} \mu \underbrace{\int_{x^{-}(h)}^{x^{+}(h)} \frac{1}{W_{0}\left(\frac{h}{e} e^{\frac{f(x)}{\varepsilon}}\right)}-\frac{1}{W_{-1}\left(\frac{h}{e} e^{\frac{f(x)}{\varepsilon}}\right)} d x}_{I_{1}(h)}
\end{aligned}
$$

It is easy to check that $h \neq-1, I_{1}(h), I_{2}(h) \neq 0$. So we can apply the implicit function theorem for finding a cycle by solving the equation $L_{+}(h, \mu, \beta)-L_{-}(h, \mu, \beta)=$ 0 . This equation is rather involved but a first approximation for it is obtained by solving

$$
\beta I_{2}(h)+\sqrt{\varepsilon} \mu I_{1}(h)=0 .
$$

This can be done using MATHEMATICA or MAPLE, where many possible computations are possible with the Lambert functions [9].

Because the homoclinic loop in the perturbed system have to be very close to the homoclinc loop in the Hamiltonian system, we solve equation (41) with the value $h=$ $e^{-\frac{1}{6 \alpha^{2} \varepsilon}}$ (level of the homoclinic loop in the Hamiltonian system). The loss of stability is found for $\mu \simeq 0.00031274$, which gives an approximation with a very high precision. 
Further developments of this observation are expected and will be the subject of a follow-up publication.

\section{References}

[1] G. Auchmuty, E. Dean, R. Glowinski, S. C. Zhang, "Control Methods for the numerical computation of periodic orbits of autonomous differential equations", in: Control Problems for Systems Described by Partial Differential Equations and Applications, I. Lasiecka and R. Triggiani Eds., Lecture Notes in Control and Information Sciences vol. 97, 1987, pp. 64-89.

[2] S. M. BAer, T. ERneuX, "Singular Hopf bifurcation to relaxation oscillations", SIAM J. Appl. Math., vol. 46, num. 5, 1986.

[3] S. M. BAER, , "Singular Hopf bifurcation to relaxation oscillations", SIAM J. Appl. Math., vol. 52, num. 6, 1992.

[4] R. Beb Bouchi, "Canards homocliniques", in: The 7th International Colloquium on Differential Equations in Bulgaria (Plovdiv), 1997.

[5] E. Benoît, "Relation entrée-sortie", C. R. Acad. Sci. Paris. Ser. I. Math., vol. 293, num. 1, 1981.

[6] E. Benoît, J.-L. Callot, F. Diener, M. Diener, "Chasse au canard", Collect. Math., vol. 32, num. 1-2, 1981.

[7] B. BRAAKSMA, "Singular Hopf bifurcation in systems with fast and slow variables", J. Nonlin. Sci., vol. 8, num. 5, 1998.

[8] L. BuŘIČ, A. Klíč, L. PurmovÁ, "Canard solutions and travelling waves in the spruce budworm population model", Appl. Math. Comput., vol. 183, num. 2, 2006.

[9] R. M. Corless, G. H. Gonnet, D. E. G. Hare, D. J. Jeffrey, D. E. Knuth, "On the Lambert W function", Adv. Comput. Math., vol. 5, num. 1, 1996.

[10] P. R. DA Silva, "Canard cycles and homoclinic bifurcation in a 3 parameter family of vector fields on the plane", Publ. Math., vol. 43, num. 1, 1999.

[11] P. De Maesschalck, M. Desroches, "Numerical Continuation Techniques for Planar Slow-Fast Systems", SIAM J. Appl. Dyn. Syst., vol. 12, num. 3, 2013.

[12] E. J. Doedel, H. B. Keller, J.-P. Kernévez, "Numerical analysis and control of bifurcation problems Part I: Bifurcation in finite dimensions", Int. J. Bifurcation and Chaos, vol. 1, num. 3, 1991.

[13] E. J. Doedel, H. B. Keller, J.-P. Kernévez, "Numerical analysis and control of bifurcation problems Part II: Bifurcation in infinite dimensions", Int. J. Bifurcation and Chaos, vol. 1, num. 4, 1991.

[14] E. J. Doedel, A. R. Champneys, F. Dercole, T. F. Fairgrieve, Y. A. Kuznetsov, B. Oldeman, R. C. Paffenroth, B. Sandstede, X. J. Wang, C. H. Zhang, "AUTO07p: Continuation and bifurcation software for ordinary differential equations", Journal, 2007, available at the URL http://indy.cs.concordia.ca. 
[15] F. Dumortier, R. Roussarie, "Birth of canard cycles", Discrete Contin. Dyn. Syst. Ser. S., vol. 2, num. 4, 2009.

[16] F. Dumortier, D. Panazzolo, R. Roussarie, "More limit cycles than expected in Liénard equations", Proc. Amer. Math. Soc., vol. 135, num. 6, 2007.

[17] W. EcKhAus, "Relaxation oscillations including a standard chase on French ducks", in: Asymptotic Analysis II, F. Verhulst Ed., Lecture Notes in Math. vol. 985, 1983.

[18] G. B. ERmentrout, , "Simulating, analyzing, and animating dynamical systems: a guide to XPPAUT for researchers and students", , vol. , num. 999, 2004.

[19] S. M. BAER, , "Article Title", Software Environment and Tools vol. 14, SIAM, Philadelphia, 2002.

[20] J.-P. Françoise, C. C. Pugh, "Keeping track of limit cycles", J. Differential Equations, vol. 65, num. 2, 1986.

[21] J.-P. Françoise, N. Roytvarf, Y. Yomdin, "Analytic continuation and fixed points of the Poincaré mapping for a polynomial Abel equation", J. Eur. Math. Soc. (JEMS), vol. 10, num. 2, 2008.

[22] R. GLOWINSKI, "Numerical methods for nonlinear variational problems", Springer Series in Computational Physics, Springer, Berlin, 1984.

[23] J. GuCKenheImer, “"Bifurcations of relaxation oscillations, in: Normal Forms, Bifurcations and Finiteness Problems in Differential Equation, Y. Ilyashenko and C. Rousseau Eds, NATO Sci. Ser. II Math. Phys. Chem vol. 137, Kluwer, Dordrecht, The Netherlands, 2004.

[24] J. GUCKENHEIMER, "Singular Hopf bifurcation in Systems with two slow variables", SIAM J. Appl. Dyn. Syst., vol. 7, num. 4, 2008.

[25] J. Guckenheimer, M. Drew LaMar, "Periodic orbit continuation in multiple time scale systems", in: Numerical continuation methods for dynamical systems: path following and boundary value problem, B. Krauskopf, H. M. Osinga and J. Galán-Vioque (Eds.), Springer, 2007.

[26] J. HÄRTERICH, "Viscous profiles of traveling waves in scalar balance laws: the canard case", Methods Appl. Anal., vol. 10, num. 1, 2003.

[27] M. Hayashi, "On canard homoclinic of a Liénard Perturbation System”, Appl. Math., vol. 2, num. 10, 2011.

[28] H. B. KeLler, "Lectures on numerical methods in bifurcation problems", Tata Institute of Fundamental Research Lectures on Mathematics and Physics vol. 79, Springer-Verlag, Berlin, 1987.

[29] M. Krupa, P. Szmolyan, "Relaxation oscillations and canard explosion", J. Differential Equations, vol. 174, num. 2, 2001.

[30] O. C. SisABER, R. BebBouChI, "On a singularly perturbed Liénard system with three equilibrium points", in: Dynamical Systems and Applications, Proceedings of a conference held in Antalya, Turkey, 5-10 July, 2004, pp. 648-651. 